\title{
Industrializing a Bacterial Strain for I-Serine Production through Translation Initiation Optimization
}

Rennig, Maja; Mundhada, Hemanshu; Wordofa, Gossa Garedew; Gerngross, Daniel; Wulff, Tune; Worberg, Andreas; Nielsen, Alex Toftgaard; Nørholm, Morten H.H.

\section{Published in:}

ACS Synthetic Biology

Link to article, DOI:

10.1021/acssynbio.9b00169

Publication date:

2019

Document Version

Peer reviewed version

Link back to DTU Orbit

Citation (APA):

Rennig, M., Mundhada, H., Wordofa, G. G., Gerngross, D., Wulff, T., Worberg, A., Nielsen, A. T., \& Nørholm, M. H. H. (2019). Industrializing a Bacterial Strain for I-Serine Production through Translation Initiation Optimization. ACS Synthetic Biology, 8(10), 2347-2358. https://doi.org/10.1021/acssynbio.9b00169

\section{General rights}

Copyright and moral rights for the publications made accessible in the public portal are retained by the authors and/or other copyright owners and it is a condition of accessing publications that users recognise and abide by the legal requirements associated with these rights.

- Users may download and print one copy of any publication from the public portal for the purpose of private study or research.

- You may not further distribute the material or use it for any profit-making activity or commercial gain

- You may freely distribute the URL identifying the publication in the public portal 


\section{Supporting information for:}

\section{Industrializing a bacterial strain for L-serine production through translation initiation optimization}

Maja Rennig ${ }^{1,3 *}$, Hemanshu Mundhada ${ }^{1 *}$, Gossa G. Wordofa ${ }^{1}$, Daniel Gerngross ${ }^{2}$, Tune Wulff ${ }^{1}$, Andreas Worberg ${ }^{1}$, Alex T. Nielsen ${ }^{1,3}$, Morten H.H. Nørholm ${ }^{1,3}$

${ }^{1}$ Novo Nordisk Foundation Center for Biosustainability, Technical University of Denmark, Kgs.

Lyngby, Denmark

${ }^{2}$ Department of Biosystems Science and Engineering, ETH Zurich, Basel, Switzerland

${ }^{3}$ Mycropt IVS, Copenhagen, Denmark

* These authors contributed equally.

Address of correspondence: morno@biosustain.dtu.dk, atn@biosustain.dtu.dk 


\section{Supplementary Figure S1.}

a

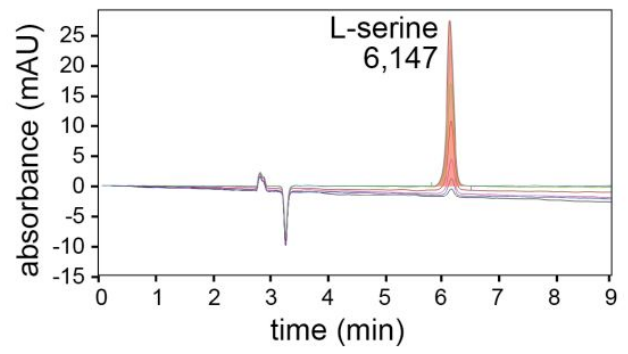

C

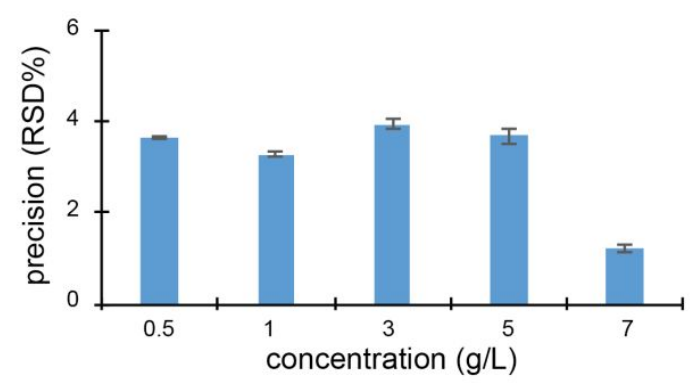

b

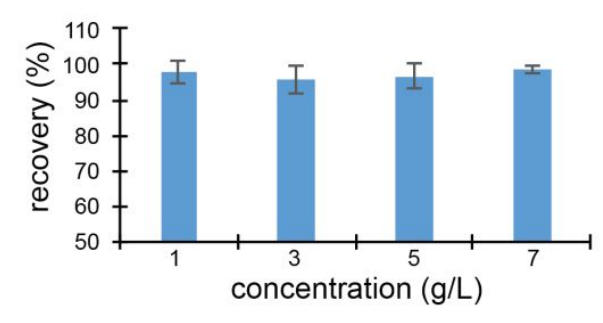

d

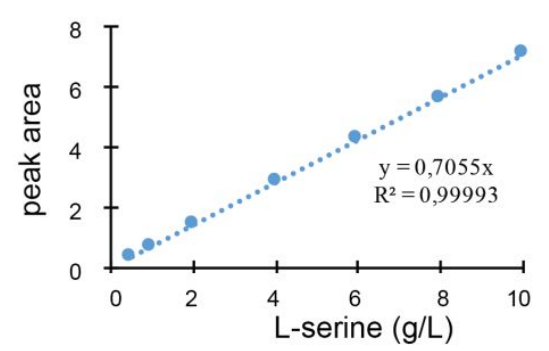

Supplementary Figure S1. Development and validation of a medium throughput HPLC method for the detection of $\mathbf{L}$-serine in microbial culture supernatant. (a) Overlaid HPLC chromatogram of L-serine standards. (b) The recovery of L-serine from spiked samples $(n=5)$. (c) Reproducability of the method ( $\mathrm{n}=5$ ) for different L-serine concentrations. (d) Calibration curve for L-serine. 


\section{Supplementary Figure S2.}

a

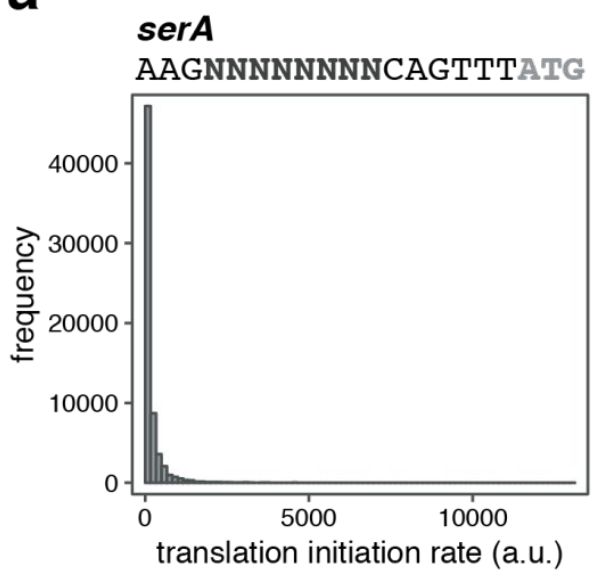

C

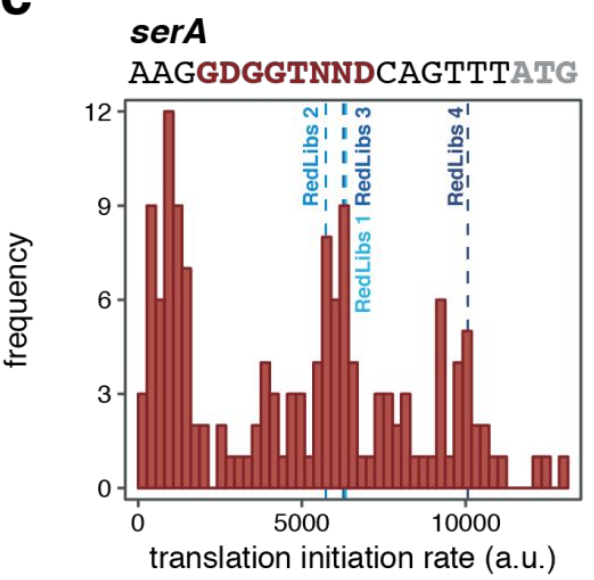

b

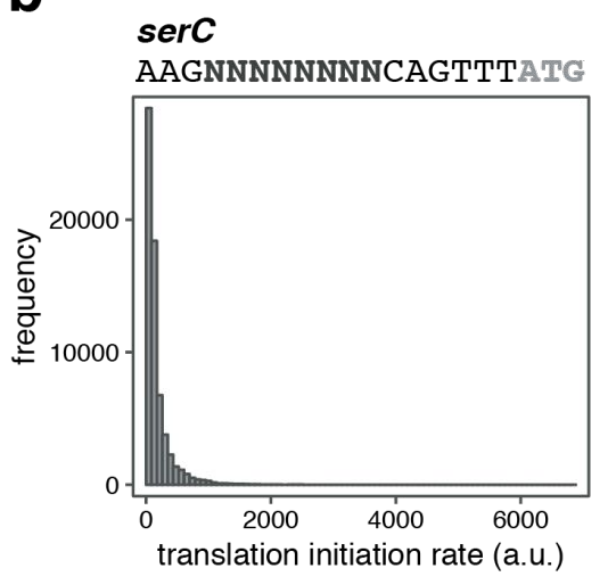

d

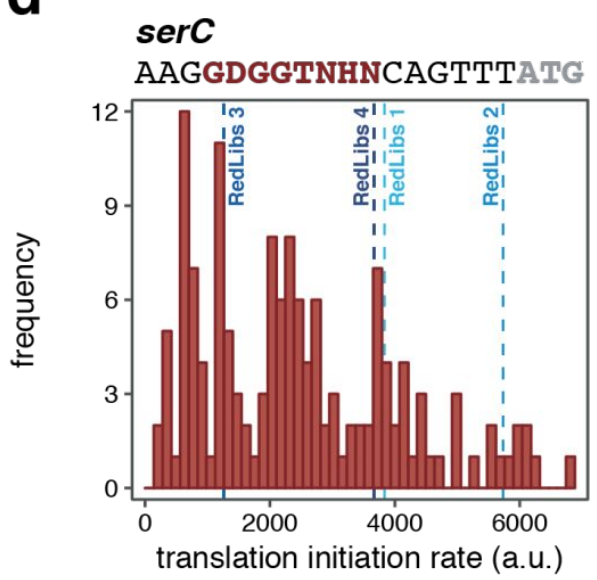

\section{Supplementary Figure S2. Translation initiation rate prediction of the input library and}

library reduced by RedLibs. Histograms of the translation initiation rate predictions for fully degenerate input libraries at the Shine-Dalgarno sequence for $\operatorname{ser} A$ (a) and $\operatorname{ser} C(\mathbf{b})$ and the output libraries reduced by RedLibs ${ }^{1}$ to $144-$ member libraries with a uniform target distribution for $\operatorname{ser} A(\mathbf{c})$ and $\operatorname{ser} C(\mathbf{d})$. In the bottom panels, dashed lines mark the translation initiation rates of the variants that were chosen for further analysis. The individual predictions for the fully degenerate input libraries were calculated using the RBS Calculator ${ }^{2}$. 


\section{Supplementary Figure S3.}

a

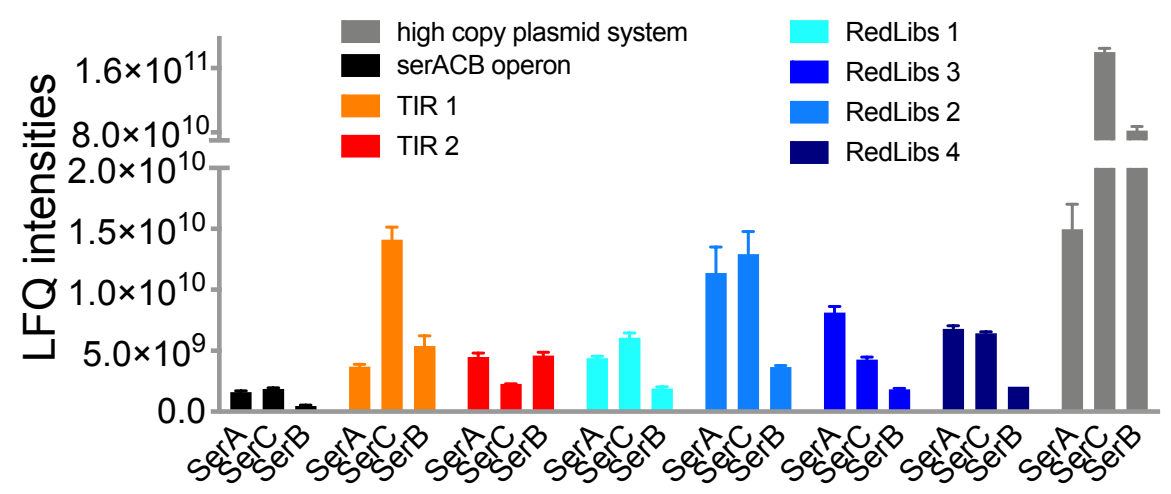

b

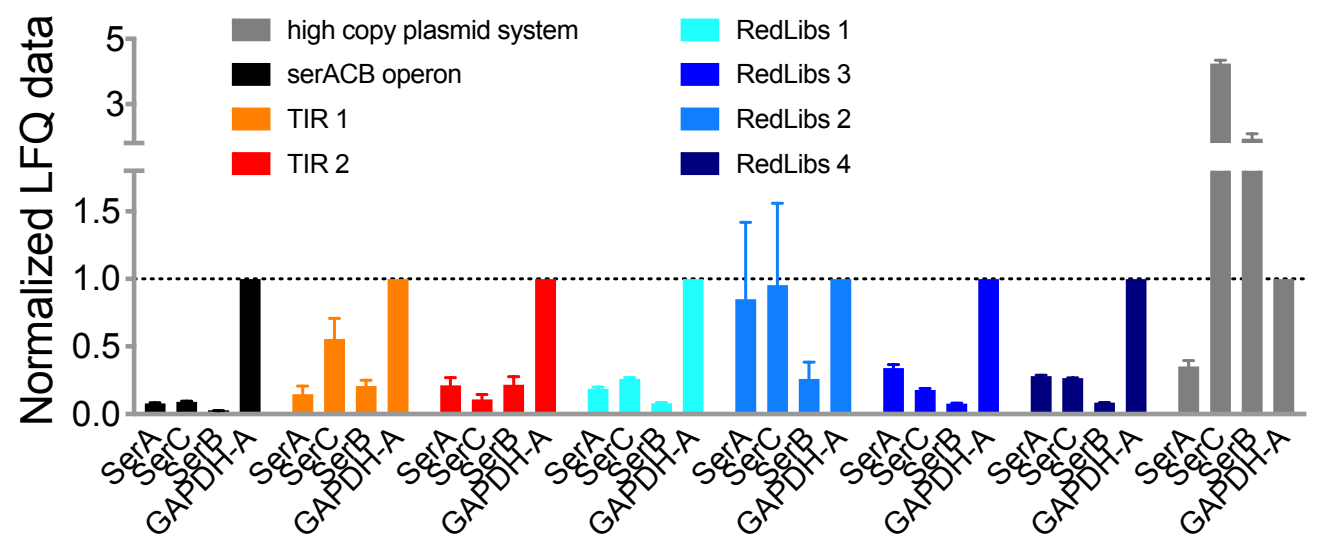

Supplementary Figure S3. Proteomics data of SerA ${ }^{\text {mut }}$, SerB and SerC. (a) LFQ intensities for the original assembled operon and all optimized variants as well as the high copy plasmid system. (b) LFQ intensities of the same sampels normalized to GAPDH-A expression. 


\section{Supplementary Figure S4.}

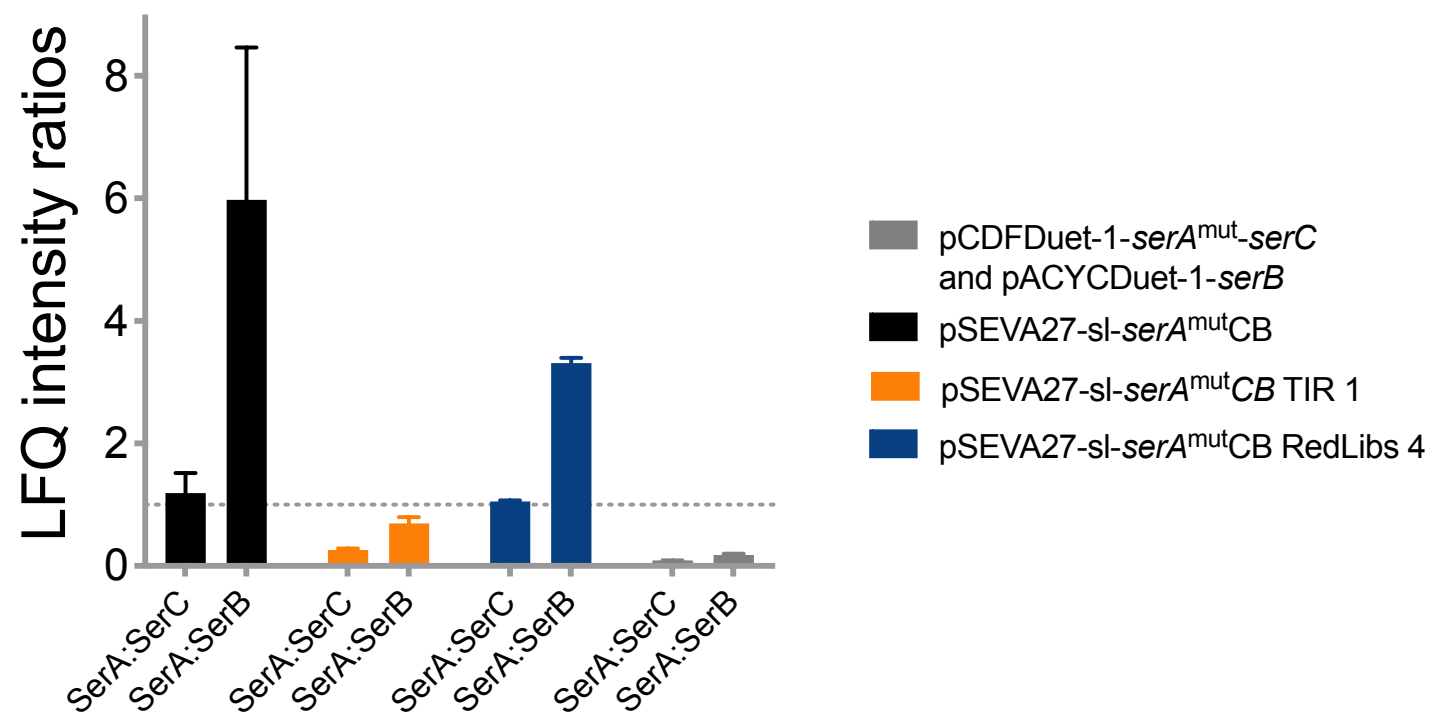

Supplementary Figure S4. Protein ratios of different $\operatorname{ser} A^{\mathrm{mut}} C B$ operons in comparison.

Ratios of SerA to SerC and SerA to SerB were calculated for the original synthetic operon pSEVA27-sl-ser $A^{\text {mut }} C B$ (black), the pSEVA27-sl-ser $A^{\text {mut }} C B$ TIR 1 operon (orange), the pSEVA27-sl-ser $A^{\text {mut }} C B$ RedLibs 4 operon (blue) and the high copy two plasmid system (grey). 


\section{Supplementary Figure S5.}

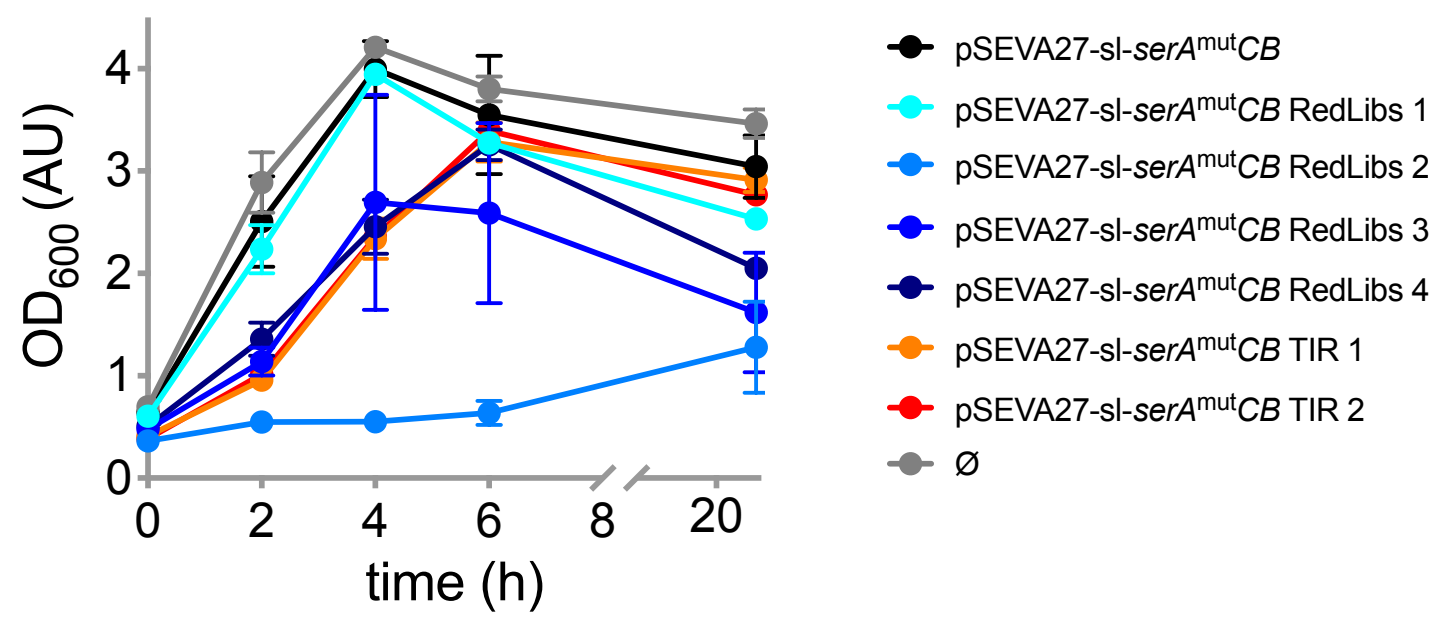

Supplementary Figure S5. Growth of all selected operons during batch fermentations.

The optical density $\left(\mathrm{OD}_{600}\right)$ of all strains was measured during a 24-hour serine production experiment. 


\section{Supplementary Figure S6.}

a

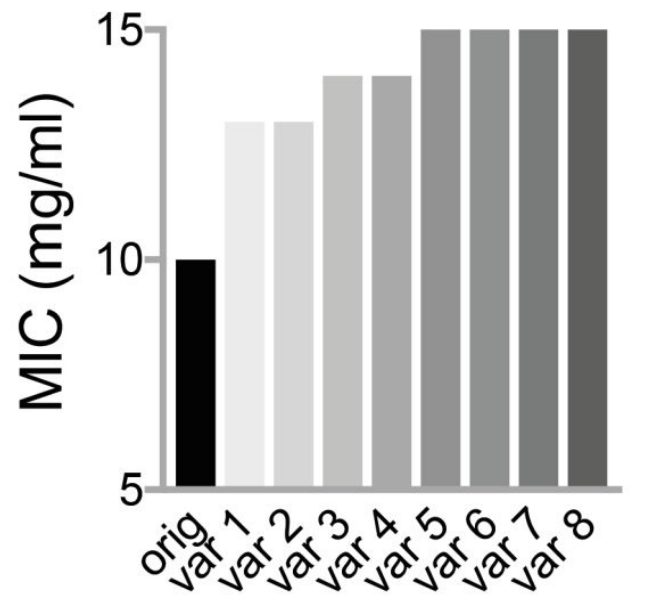

b

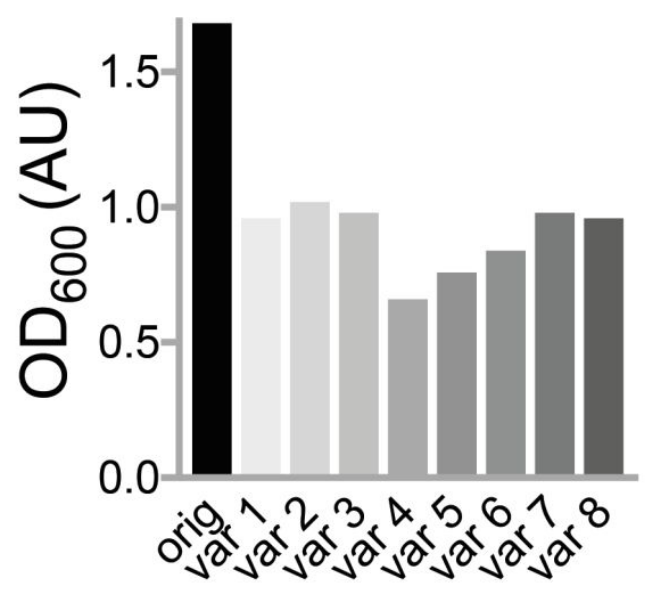

Supplementary Figure S6. Assessment of effects of expression optimization of $\operatorname{ser} C$-hpbla. Expression of $\operatorname{ser} C$ was translationally coupled to the ampicillin resistance gene bla. Expression levels of the original construct (orig) and selected expression library variants (var 1-8) were estimated by resistance to ampicillin (a). The optical density of cultures expressing the original construct or a selected library variant was analysed after 20 hours of expression in the absence of ampicillin (b). 



\section{Supplementary Table S1.}

\section{Supplementary Table S1. Strains used in this study}

\begin{tabular}{|c|c|c|}
\hline Strain & Genotype & Source/Reference \\
\hline E. coli $\mathrm{NEB} 5 \alpha$ & $\begin{array}{l}\text { fhuA2 } \Delta(\operatorname{argF}-1 \text { acZ }) \text { U169 phoA glnV44 } \Phi 80 \Delta(\text { lacZ)M15 } \\
\text { gyrA96 recA1 relA1 endA1 thi-1 hsdR17 }\end{array}$ & $a$ \\
\hline E. coli $\mathrm{MC} 1061$ & $\begin{array}{l}\operatorname{araD} 139, \Delta(\operatorname{ara}, \text { leu }) 7697, \Delta \text { lacX74, galU-, galK-, hsr-, } \\
\text { hsm+, strA }\end{array}$ & In house \\
\hline E. coli BL21(DE3) & $\mathrm{F}^{-}$ompT hsdS $S_{\mathrm{B}}\left(\mathrm{r}_{\mathrm{B}}^{-} \mathrm{m}_{\mathrm{B}}^{-}\right)$gal dcm (DE3) & $b$ \\
\hline E. coli ALE-5(DE3) & MG1655 $\Delta s d a A \Delta s d a B \Delta t d c G \Delta g l y A$ thr $A^{\text {mut }} \lambda(\mathrm{DE} 3)$ & 3 \\
\hline $\begin{array}{l}\text { E. coli ALE-5(DE3) } \\
\operatorname{ser}^{\text {mut }} C B \text { TIR1 }\end{array}$ & $\begin{array}{l}\text { MG1655 } \Delta s d a A \Delta s d a B \Delta t d c G \Delta g l y A \text { thr } A^{\text {mut }} \text { glsmS- } \\
\text { pstS::ser } A^{\text {mut } C B} \lambda(\mathrm{DE} 3)\end{array}$ & This study \\
\hline
\end{tabular}




\section{Supplementary Table S2.}

\section{Supplementary Table S2. Plasmids used in this study}

\begin{tabular}{|c|c|c|}
\hline Plasmid & Property & Source/Reference \\
\hline pCDFDuet-1-serAmut-serC & vector encoding $\operatorname{ser} A$ and $\operatorname{ser} C, \mathrm{Sp}^{\mathrm{R}}$ & 3 \\
\hline pACYCDuet-1-serB & vector encoding $\operatorname{ser} B, \mathrm{Cm}^{\mathrm{R}}$ & 3 \\
\hline pSEVA27-sl3-narK-gfp & $\begin{array}{l}\text { vector encoding nark- } g f p, \mathrm{Km}^{\mathrm{R}} \text {, used to } \\
\text { amplify pSEVA } 27 \text {-sl backbone }\end{array}$ & 4 \\
\hline pACYC-dashergfp-hp-bla & $\begin{array}{l}\text { vector encoding dashergfp translationally } \\
\text { coupled to bla, } \mathrm{Cm}^{\mathrm{R}} \text {, used to amplify } \\
\text { hp-bla }\end{array}$ & 5 \\
\hline pSEVA27-sl3-serAmut-hp-bla & $\begin{array}{l}\text { vector encoding } \operatorname{ser} A \text { translationally } \\
\text { coupled to bla, } \mathrm{Km}^{\mathrm{R}}\end{array}$ & This study \\
\hline $\begin{array}{l}\text { pSEVA27-s13-serAmut-serCB-hp- } \\
\text { bla }\end{array}$ & $\begin{array}{l}\text { vector encoding a synthetic operon } \\
\text { ser } A C B \text { translationally coupled to bla, } \\
\mathrm{Km}^{\mathrm{R}}\end{array}$ & This study \\
\hline $\begin{array}{l}\text { pSEVA27-sl3-serAmut-opt-serCB- } \\
\text { hp-bla }\end{array}$ & $\begin{array}{l}\text { vector encoding an optimized synthetic } \\
\text { operon } \operatorname{ser} A C B \text { translationally coupled to } \\
\text { bla, } \mathrm{Km}^{\mathrm{R}}\end{array}$ & This study \\
\hline $\begin{array}{l}\text { pSEVA27-sl3-serAmut-opt- } \\
\text { serCBopt-hp-bla }\end{array}$ & $\begin{array}{l}\text { vector encoding an optimized synthetic } \\
\text { operon } \operatorname{ser} A C B \text { translationally coupled to } \\
\text { bla, } \mathrm{Km}^{\mathrm{R}}\end{array}$ & This study \\
\hline $\begin{array}{l}\text { pSEVA27-s13-serAmut-serCB-hp- } \\
\text { bla RedLibs1 }\end{array}$ & $\begin{array}{l}\text { vector encoding an optimized synthetic } \\
\text { operon } \operatorname{ser} A C B \text { translationally coupled to } \\
\text { bla, } \mathrm{Km}^{\mathrm{R}}\end{array}$ & This study \\
\hline $\begin{array}{l}\text { pSEVA27-s13-serAmut-serCB-hp- } \\
\text { bla RedLibs2 }\end{array}$ & $\begin{array}{l}\text { vector encoding an optimized synthetic } \\
\text { operon } \operatorname{ser} A C B \text { translationally coupled to } \\
\text { bla, } \mathrm{Km}^{\mathrm{R}}\end{array}$ & This study \\
\hline $\begin{array}{l}\text { pSEVA27-s13-serAmut-serCB-hp- } \\
\text { bla RedLibs } 3\end{array}$ & $\begin{array}{l}\text { vector encoding an optimized synthetic } \\
\text { operon } \operatorname{ser} A C B \text { translationally coupled to } \\
\text { bla, } \mathrm{Km}^{\mathrm{R}}\end{array}$ & This study \\
\hline $\begin{array}{l}\text { pSEVA27-s13-serAmut-serCB-hp- } \\
\text { bla RedLibs4 }\end{array}$ & $\begin{array}{l}\text { vector encoding an optimized synthetic } \\
\text { operon } \operatorname{ser} A C B \text { translationally coupled to } \\
\text { bla, } \mathrm{Km}^{\mathrm{R}}\end{array}$ & This study \\
\hline pSEVA27-sl3-serAmut-serCB & $\begin{array}{l}\text { vector encoding a synthetic operon } \\
\operatorname{ser} A C B, \mathrm{Km}^{\mathrm{R}}\end{array}$ & This study \\
\hline pSEVA27-sl3-serAmut-opt-serCB & $\begin{array}{l}\text { vector encoding an optimized synthetic } \\
\text { operon } \operatorname{ser} A C B, \mathrm{Km}^{\mathrm{R}}\end{array}$ & This study \\
\hline $\begin{array}{l}\text { pSEVA27-sl3-serAmut-opt- } \\
\text { serCBopt }\end{array}$ & $\begin{array}{l}\text { vector encoding an optimized synthetic } \\
\text { operon } \operatorname{ser} A C B, \mathrm{Km}^{\mathrm{R}}\end{array}$ & This study \\
\hline $\begin{array}{l}\text { pSEVA27-s13-serAmut-serCB } \\
\text { RedLibs1 }\end{array}$ & $\begin{array}{l}\text { vector encoding an optimized synthetic } \\
\text { operon } \operatorname{ser} A C B, \mathrm{Km}^{\mathrm{R}}\end{array}$ & This study \\
\hline $\begin{array}{l}\text { pSEVA27-s13-serAmut-serCB } \\
\text { RedLibs2 }\end{array}$ & $\begin{array}{l}\text { vector encoding an optimized synthetic } \\
\text { operon } \operatorname{ser} A C B, \mathrm{Km}^{\mathrm{R}}\end{array}$ & This study \\
\hline $\begin{array}{l}\text { pSEVA27-s13-serAmut-serCB } \\
\text { RedLibs3 }\end{array}$ & $\begin{array}{l}\text { vector encoding an optimized synthetic } \\
\text { operon } \operatorname{ser} A C B, \mathrm{Km}^{\mathrm{R}}\end{array}$ & This study \\
\hline $\begin{array}{l}\text { pSEVA27-s13-serAmut-serCB } \\
\text { RedLibs4 }\end{array}$ & $\begin{array}{l}\text { vector encoding an optimized synthetic } \\
\text { operon } \operatorname{ser} A C B, \mathrm{Km}^{\mathrm{R}}\end{array}$ & This study \\
\hline
\end{tabular}




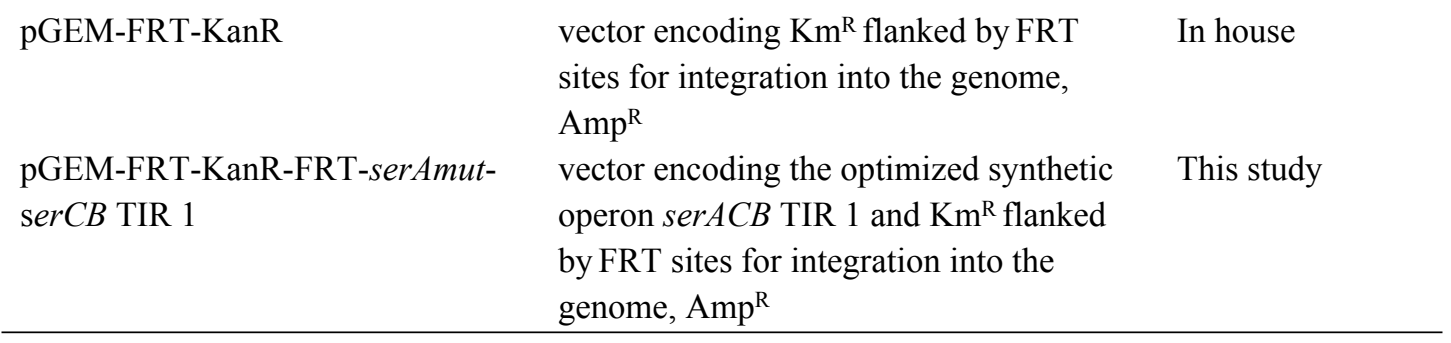

\section{Supplementary Table S3.}

Supplementary Table S3. Oligonucleotides used in this study

\begin{tabular}{|c|c|c|}
\hline No. & Name & Sequence $\left(5^{\prime}-->3^{\prime}\right)$ \\
\hline & \multicolumn{2}{|c|}{ Cloning oligonucleotides } \\
\hline 1 & pSEVA27_fwd & AGCTGAGGUCGCCTCAGC \\
\hline 2 & pSEVA27_T7_rev & $\begin{array}{l}\text { AAACTGGTCUCCTTCTTAAAGTTAAACAAAATTATTTCTAGA } \\
\text { G }\end{array}$ \\
\hline 3 & SerA_fwd & AGACCAGTTUATGGCAAAGGTATCGC \\
\hline 4 & SerA_rev & ACCTCCTAUGTCAGTACAGCAGACG \\
\hline 5 & hp_Bla_fwd & ATAGGAGGUCCTCCTATGTCAATTCAACATTTCCGTGTC \\
\hline 6 & hp_Bla_rev & ACCUCAGCUTACCAATGCTTAATCAGTG \\
\hline 7 & SerA-C_rev & ACCCGGAAUAGCTTTCATTGCCTGC \\
\hline 8 & SerA-C_fwd & ATTCCGGGUACCATTCGCGCCCGTCTGCTGTACTGATAAAA \\
\hline & & GAAGGAGACCAGTTTATGGCTCAAATCTTCAATTTTAGTTC \\
\hline 9 & SerC-B_rev & $\begin{array}{l}\text { ATCAACCGUGACGGCGTTCGAACTCAACCATGAAGTCTGTC } \\
\text { AGCG }\end{array}$ \\
\hline 10 & SerC-B_fwd & $\begin{array}{l}\text { ACGGTTGAUAAAAGAAGGAGACCAGTTTATGCCTAACATTA } \\
\text { CCTG }\end{array}$ \\
\hline 11 & SerB_rev & ACСTCCTAUGTCACTTCTGATTCAGGC \\
\hline 12 & SerA_lib_rev & $\begin{array}{l}\text { TCTCСTTCTTAAAGTTAAACAAAATTATTCTAGAGGGGAATT } \\
\text { GTTATCCG }\end{array}$ \\
\hline 13 & SerA_TIRlib_fwd & $\begin{array}{l}\text { ACTTTAAGAAGGAGACNNNNNNATGGCNAARGTATCGCTGG } \\
\text { AG }\end{array}$ \\
\hline 14 & SerB_TIRlib_fwd & $\begin{array}{l}\text { ACGGTTGAUAAAAGAAGGAGACNNNNNNATGCCNAAYATT } \\
\text { ACCTG }\end{array}$ \\
\hline 15 & SerC_TIRlib_fwd & $\begin{array}{l}\text { ATTCCGGGUACCATTCGCGCCCGTCTGCTGTACTGATAAAA } \\
\text { GAAGGAGACNNNNNNATGGCNCARATCTTCAATTTTAGTTC }\end{array}$ \\
\hline 16 & SerA_RedLibs_fwd & $\begin{array}{l}\text { AATTTTGTTUAACTTTAAGGDGGTNNDCAGTTTATGGCAAA } \\
\text { GGTATCGCTGGA }\end{array}$ \\
\hline 17 & SerC_RedLibs_fwd & ATCTTCAATUTTAGTTCTGGTCCGGCA \\
\hline 18 & SerC_RedLibs_rev & $\begin{array}{l}\text { AATTGAAGAUTTGAGCCATAAACTGNDNACCHCCTTTTATC } \\
\text { AGTACAGCAGACGGGCGCGAA }\end{array}$ \\
\hline 19 & SEVA27_BB_rev & AAACAAAATUATTCTAGAGGGGAATTGTTATCCG \\
\hline 20 & Bla_remove_fwd & AGCTGAGGUCGCCTCAGCGGCCGGCCC \\
\hline 21 & Bla_remove_rev & ACCTCAGCUTTACTTCTGATTCAGGCTGCCTG \\
\hline 22 & $\begin{array}{l}\text { SerACB_in_pGEM_ } \\
\text { fwd }\end{array}$ & АСТTCСТCAUTTGTCCTACTCAGGAGAG \\
\hline 23 & $\begin{array}{l}\text { SerACB_in_pGEM_ } \\
\text { rev }\end{array}$ & ATTTTCTCCUATTCTCACCAATAAAAAACGCCCG \\
\hline 24 & pGEM_BB_fwd & AGGAGAAAAUTGCGTTGCGCTCACTGC \\
\hline
\end{tabular}


25 pGEM_BB_rev

26 Genome_int_fwd

27 Genome_int_rev
ATGAGGAAGUTCCTATACTTTCTAGAGAATAGGAACTTCCG CGCCGCACACA

GCGTAACCTGGCAAAATCGGTTACGGTTGAGTAATAAATGG ATACTAGAGAATTAACCCTCACTAAAGGGCG

TTACAAACATTAATAACGAAGAGATGACAGAAAAATTTTCA TTCTGTGACATTCTCACCAATAAAAAACG

\begin{tabular}{lll}
\hline \multicolumn{3}{c}{ Seqeuncing oligonucleotides } \\
\hline 28 & SerA_seq_fwd & CTCAGGTACAGCATCTTTCTG \\
29 & SerA_seq_rev & GTACATGCAGACTCACCACATCG \\
30 & SerC_seq_fwd & GGTCGCCGCTGACTTCTCTTC \\
31 & SerC_seq_rev & CACCATAACGGCTGACGTCAA \\
32 & SerB_seq_fwd & GGAAGTAACCGAACGGGCGATG \\
33 & SerB_seq_rev & CAGCGTCGCCACACGGCTGCG \\
34 & glmS-pstS_fwd & GGCTTACCATGTCGCGCTGATCA \\
35 & glmS-pstS_rev & CGCGGCGACAACAGTTGCGAC
\end{tabular}




\section{Supplementary Table S4.}

Supplementary Table S4. Sequences of original clones and optimized TIR and RBS library variants

\begin{tabular}{|c|c|c|}
\hline Gene /Operon name & Sequence & Comments \\
\hline \multicolumn{3}{|c|}{ A. TIR variants of individual $\operatorname{ser} A^{\text {mut }}$ gene } \\
\hline 1. $\operatorname{ser} A^{\text {mut }}$ & 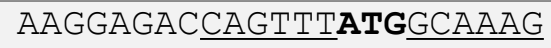 & \\
\hline $\operatorname{ser} A^{\text {mut }}$ opt & AAGGAGACTGATCTATGGCGAAA & variant 7 and 8 \\
\hline \multicolumn{3}{|c|}{ B. TIR variants of ser $A^{\text {mut }}$ opt-serCB operon } \\
\hline \multicolumn{3}{|c|}{ 1. Original operon } \\
\hline $\operatorname{ser} A^{\text {mut }}$ & 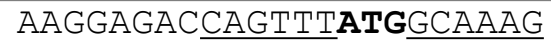 & \multirow{3}{*}{ pSEVA27-sl-ser $A^{\text {mut }} C B$} \\
\hline $\operatorname{serC}$ & AAGGAGACCAGTTTATGGCTCAA & \\
\hline serB & AAGGAGACCAGTTTATGCCTAAC & \\
\hline \multicolumn{3}{|l|}{ 2. Operon 1} \\
\hline $\operatorname{ser} A^{\text {mut }}$ & AAGGAGACTGATCTATGGCGAAA & \multirow{3}{*}{ pSEVA27-sl-ser $A^{\text {mut }} C B$ TIR 1} \\
\hline serC & AAGGAGACCAGTTTATGGCTCAA & \\
\hline serB & AAGGAGACCAGTTTATGCCTAAC & \\
\hline \multicolumn{3}{|l|}{ 3. Operon 2} \\
\hline $\operatorname{ser} A^{\text {mut }}$ & AAGGAGACTGATCTATGGCGAAA & \multirow{3}{*}{ pSEVA27-sl-ser $A^{\text {mut }} C B$ TIR 2} \\
\hline serC & AAGGAGACCAGTTTATGGCTCAA & \\
\hline $\operatorname{ser} B$ & AAGGAGACTGACTAATGCCGAAT & \\
\hline \multicolumn{3}{|c|}{ C. RBS variants of $\operatorname{ser} A^{\mathrm{mut}} \operatorname{ser} C B$ operon } \\
\hline \multicolumn{3}{|l|}{ 1. Operon 1} \\
\hline $\operatorname{ser} A^{\text {mut }}$ & GGGGTAGGCAGTTTATGGCAAAG & \multirow{3}{*}{ pSEVA27-sl-ser $A^{\text {mut }} C B$ RedLibs 1} \\
\hline $\operatorname{serC}$ & GAGGTAACCAGTTTATGGCTCAA & \\
\hline $\operatorname{ser} B$ & AAGGAGACCAGTTTATGCCTAAC & \\
\hline \multicolumn{3}{|l|}{ 2. Operon 2} \\
\hline $\operatorname{ser} A^{\text {mut }}$ & GGGGTGGGCAGTTTATGGCAAAG & \multirow{3}{*}{ pSEVA27-sl-ser $A^{\text {mut }} C B$ RedLibs 2} \\
\hline $\operatorname{serC}$ & GAGGTAAACAGTTTATGGCTCAA & \\
\hline $\operatorname{ser} B$ & AAGGAGACCAGTTTATGCCTAAC & \\
\hline \multicolumn{3}{|l|}{ 3. Operon 3} \\
\hline $\operatorname{ser} A^{\text {mut }}$ & GGGGTGTGCAGTTTATGGCAAAG & \multirow{3}{*}{ pSEVA27-sl-ser $A^{\text {mut }} C B$ RedLibs 3} \\
\hline $\operatorname{serC}$ & GGGGTGTGCAGTTTATGGCTCAA & \\
\hline $\operatorname{ser} B$ & 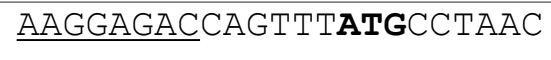 & \\
\hline \multicolumn{3}{|l|}{ 4. Operon 4} \\
\hline $\operatorname{ser} A^{\text {mut }}$ & GAGGTGTGCAGTTTATGGCAAAG & \multirow{2}{*}{ pSEVA27-sl-ser $A^{\text {mut }} C B$ RedLibs 4} \\
\hline $\operatorname{serC}$ & GAGGTCACCAGTTTATGGCTCAA & \\
\hline
\end{tabular}


Supplementary Table S5.

\section{Supplementary Table S5. LFQ data}

\begin{tabular}{|c|c|c|c|c|}
\hline Operon/protein & \multicolumn{2}{|c|}{ LFQ intensity } & Mean & SD \\
\hline \multicolumn{5}{|l|}{ pSEVA27-sl-ser $A^{\text {mut }} C B$} \\
\hline SerA & $1,684,300,000$ & $1,537,500,000$ & $1,610,900,000$ & $103,803,275.5$ \\
\hline SerC & $1,926,000,000$ & $1,813,600,000$ & $1,869,800,000$ & $79,478,802.21$ \\
\hline SerB & $418,160,000$ & $513,240,000$ & $465,700,000$ & $67,231,712.76$ \\
\hline GAPDH & $20,366,000,000$ & $20,626,000,000$ & $20,496,000,000$ & $183,847,763.1$ \\
\hline \multicolumn{5}{|c|}{ pSEVA27-sl-ser $A^{\text {mut }} C B$ TIR 1} \\
\hline SerA & $3,828,200,000$ & $3,568,800,000$ & $3,698,500,000$ & $183,423,499.0$ \\
\hline SerC & $13,410,000,000$ & $14,857,000,000$ & $14,133,500,000$ & $1,023,183,512.4$ \\
\hline SerB & $4,805,600,000$ & $5,978,700,000$ & $5,392,150,000$ & $829,506,965.0$ \\
\hline GAPDH & $20,214,000,000$ & $33,060,000,000$ & $26,637,000,000$ & $9,083,493,711$ \\
\hline \multicolumn{5}{|c|}{ pSEVA27-sl-ser $A^{\text {mut }} C B$ TIR 2} \\
\hline SerA & $4,715,500,000$ & $4,286,000,000$ & $4,500,750,000$ & $303,702,362.5$ \\
\hline SerC & $2,269,100,000$ & $2,265,200,000$ & $2,267,150,000$ & $2,757,716.4$ \\
\hline SerB & $4,792,700,000$ & $4,401,500,000$ & $4,597,100,000$ & $276,620,172.8$ \\
\hline GAPDH & $27,558,000,000$ & $16,931,000,000$ & $22,244,500,000$ & $7,514,423,764$ \\
\hline pSEVA27-sl-ser $A^{\text {mut }} C$ & ILibs 1 & & & \\
\hline SerA & $4,271,600,000$ & $4,496,700,000$ & $4,384,150,000$ & $159,169,736.4$ \\
\hline SerC & $6,349,100,000$ & $5,783,200,000$ & $6,066,150,000$ & $400,151,727.5$ \\
\hline SerB & $2,003,300,000$ & $1,824,600,000$ & $1,913,950,000$ & $126,359,981.8$ \\
\hline GAPDH & $23,726,000,000$ & $22,918,000,000$ & $23,322,000,000$ & $571,342,279$ \\
\hline pSEVA27-sl-ser $A^{\text {mut }} C$ & Libs 2 & & & \\
\hline SerA & $9,888,000,000$ & $12,890,000,000$ & $11,389,000,000$ & $2,122,734,557.1$ \\
\hline SerC & $11,619,000,000$ & $14,238,000,000$ & $12,928,500,000$ & $1,851,912,660$ \\
\hline SerB & $3,753,200,000$ & $3,579,700,000$ & $3,666,450,000$ & $122,683,026.5$ \\
\hline GAPDH & $21,960,000,000$ & $10,298,000,000$ & $16,129,000,000$ & $8,246,279,282$ \\
\hline pSEVA27-sl-ser $A^{\mathrm{mut}} C$ & Libs 3 & & & \\
\hline SerA & $7,770,200,000$ & $8,497,200,000$ & $8,133,700,000$ & $514,066,629.9$ \\
\hline SerC & $4,161,900,000$ & $4,419,600,000$ & $4,290,750,000$ & $182,221,417.5$ \\
\hline SerB & $1,792,600,000$ & $1,883,100,000$ & $1,837,850,000$ & $63,993,163.7$ \\
\hline GAPDH & 24228000000 & 23696000000 & 23962000000 & $376,180,808$ \\
\hline pSEVA27-sl-ser $A^{\text {mut }} C$ & Libs 4 & & & \\
\hline SerA & $6,972,600,000$ & $6,631,300,000$ & $6,801,950,000$ & $241,335,544.4$ \\
\hline SerC & $6,513,200,000$ & $6,378,900,000$ & $6,446,050,000$ & $94,964,440.7$ \\
\hline SerB & $2,052,700,000$ & $2,052,300,000$ & $2,052,500,000$ & $282,842.7$ \\
\hline GAPDH & $24,392,000,000$ & $24,046,000,000$ & $24,219,000,000$ & $244,658,946$ \\
\hline pCDFDuet-1-ser $A^{\text {mut }_{-}}$ & and pACYCDuet & & & \\
\hline SerA & $13,649,000,000$ & $17,337,000,000$ & $14,987,333,333$ & $2,041,428,993$ \\
\hline SerC & $176,040,000,000$ & $184,470,000,000$ & $180,306,666,666.7$ & $4,215,949,873$ \\
\hline
\end{tabular}




\begin{tabular}{|c|c|c|c|c|c|}
\hline SerB & $78,093,000,000$ & $87,967,000,000$ & $80,839,000,000$ & $82,299,666,667$ & $5,096,482,055$ \\
\hline GAPDH & $42,486,000,000$ & $41,690,000,000$ & $43,103,000,000$ & $42,426,333,333$ & $708,387,135$ \\
\hline
\end{tabular}




\section{References}

(1) Jeschek, M., Gerngross, D., and Panke, S. (2016) Rationally reduced libraries for combinatorial pathway optimization minimizing experimental effort. Nat. Commun. 7, 11163. (2) Salis, H. M., Mirsky, E. A., and Voigt, C. A. (2009) Automated design of synthetic ribosome binding sites to control protein expression. Nat. Biotechnol. 27, 946-950.

(3) Mundhada, H., Seoane, J. M., Schneider, K., Koza, A., Christensen, H. B., Klein, T., Phaneuf, P. V., Herrgard, M., Feist, A. M., and Nielsen, A. T. (2017) Increased production of L-serine in Escherichia coli through Adaptive Laboratory Evolution. Metab. Eng. 39, 141150.

(4) Kim, S. H., Cavaleiro, A. M., Rennig, M., and Nørholm, M. H. H. (2016) SEVA linkers: a versatile and automatable DNA backbone exchange standard for synthetic biology. ACS Synth. Biol. 5, 1177-1181.

(5) Rennig, M., Martinez, V., Mirzadeh, K., Dunas, F., Röjsäter, B., Daley, D. O., and Nørholm, M. H. H. (2018) TARSyn: Tunable Antibiotic Resistance Devices Enabling Bacterial Synthetic Evolution and Protein Production. ACS Synth. Biol. 7, 432-442. 